\title{
La diversité linguistique et culturelle à l'école primaire : quels enjeux pour les enseignants de La Dominique?
}

\author{
Stella CAMBRONE-LASNES \\ ESPE de l'Université de Franche-Comté - ELLIADD, EA 4661
}

\section{Résumé}

La république de la Dominique présente une situation sociolinguistique particulière où conflit et contact de langues (anglais, garifuna, kokoy, français, espagnol, chinois, hindi, créole haïtien et/ou créole dominiquais) rythment la vie quotidienne des locuteurs. Dans cet article, nous proposons d'interroger la manière dont la prise en compte scolaire de cette diversité linguistique et culturelle peut transformer le rôle des enseignants et, par conséquent, leur formation. Comment l'école peut-elle favoriser un meilleur apprentissage du vivre ensemble ? Et plus globalement encore, quel genre de citoyens la Dominique souhaite-t-elle forger à l'ère de la mondialisation ? Pour mener à bien cette recherche-action de type exploratoire, nous proposons, à travers une enquête par questionnaire et par entretien semi-directif menée auprès des enseignants, d'analyser leurs discours (épilinguistiques et métalinguistiques) et représentations des langues. Les premiers résultats mettent en évidence quelques indicateurs de l'émergence et du développement d'une éducation au plurilinguisme en contexte scolaire dominiquais à travers des activités d'éveil aux langues.

\section{Mots-clés}

Plurilinguisme, représentations, éveil aux langues, école primaire, formation professionnelle.

\begin{abstract}
The Commonwealth of Dominica presents a particular sociolinguistic situation where language conflict and contact (English, Garifuna, Kokoy, French, Spanish, Chinese, Hindi, Haitian Creole and/or Dominican French Creole) give rhythm to the speakers' everyday life. In this paper, we suggest questioning the way the school consideration of this linguistic and cultural diversity can transform teachers' role and, consequently, their training. How can the school favour a better learning of living together? And more globally still, what kind of citizens Dominica wish to forge in the era of globalization? To bring this action-research of exploratory type to a successful conclusion, we suggest, through a survey by questionnaire and semi-directive interview led with teachers, analysing their (epilinguistic and metalinguistic) speeches and attitudes towards languages. The first results highlight some indicators of the emergence and the development of a plurilingual education in the Dominican school context through language awakening activities.
\end{abstract}

\section{Keywords}

Plurilingualism, attitudes, language awakening, primary education, teacher training. 


\section{Introduction}

Le sociologue Peter Ludwig Berger (1997) explique dans son essai intitulé Four faces of global culture que toutes les langues portent en elles un ensemble de valeurs, de sensibilités, d'approches de la réalité, qui toutes s'insinuent dans la conscience de ceux qui les parlent. Du point de vue de l'auteur, il est logique d'assumer que l'attrait de l'anglais, et particulièrement de sa forme américaine, est dû, au moins en partie, à sa capacité à exprimer les sensibilités d'un monde dynamique, pluraliste et rationnellement innovant. À l'instar de cette grille de lecture sociologique des effets linguistiques de la mondialisation (uniformisation des modes de communication avec l'anglais qui devient LA langue internationale), on voit apparaitre un intérêt grandissant pour l'étude de la diversité linguistique et culturelle en contexte scolaire (Cambrone-Lasnes, Kruger et Thamin, à paraître; Hélot et Rubio, 2013). En effet, l'institution qu'est l'école a un rôle significatif à jouer dans la valorisation et la promotion de la diversité linguistique et culturelle comme le démontrent les travaux de Diana-Lee Simon et Marie-Odile Maire Sandoz (2008 : 266-267) sur l'école du plurilinguisme :

"L'école n'a-t-elle pas [...] comme mission de doter les élèves d'atouts leur permettant de s'inscrire socialement dans une pluralité complexe [...] ? L'institution scolaire a vocation à accueillir tous les élèves quelle que soit leur origine culturelle et linguistique, et à ouvrer à l'inclusion et à la cohésion sociale dans une configuration mêlant des cultures endogènes et exogènes (Dabène 1989 et 1994). [...] Il y a lieu de s'interroger sur la manière dont elle peut prendre en compte l'identité de l'élève dans toute sa complexité, et comment elle peut l'ouvrir à l'altérité, à la pluralité, tout en assurant la transmission d'un ensemble de références collectives [...] dans une visée de développement de capacités plurilingues et pluriculturelles, posées ici comme facteur de réussite pour tous les élèves. »

En nous associons à cette dynamique de pensée, notre étude du terrain dominiquais cherche à dépasser les réticences ${ }^{1}$ des acteurs scolaires à tout projet d'enseignement bilingue anglaiscréole en contournant l'étude classique des situations de diglossie (langue haute " anglais » versus langue basse « créole ») et les débats stériles qui y sont associés. Notre recherche vise à interroger une approche plurielle des langues et des cultures par une analyse des discours et des représentations des enseignants vis-à-vis des langues afin d'identifier d'une part, les enjeux sous-jacents à une éducation au plurilinguisme à la Dominique, et d'autre part, de mesurer la pertinence d'une mise en œuvre d'activités d'éveil aux langues en contexte dominiquais. À ce jour, aucune recherche d'envergure n'a été menée en ce sens. Après avoir travaillé sur les pratiques langagières dans les Antilles françaises (Cambrone, 2008) et étant originaire de la Dominique, c'est tout naturellement que nous avons fait le choix ici de circonscrire notre recherche à cette zone géographique encore peu explorée et exploitée (Cambrone-Lasnes, à paraître ; Paugh, 2005 ; Bryan et Burnette, 2003).

La présente contribution s'articulera en quatre étapes :

- Un bref rappel de ce qu'est l'éveil aux langues de manière générale, en particulier les modalités de cette approche pédagogique en contexte créolophone.

- Une présentation du «macro-contexte » de la recherche afin de comprendre la dynamique sociale et culturelle des langues en présence à la Dominique, la structure et le fonctionnement du système éducatif et la politique linguistique éducative qui en découle.

\footnotetext{
${ }^{1}$ Compte tenu des moyens matériels et financiers à mobiliser et des représentations dévalorisantes vis-à-vis du créole.
} 
- Une information sur les outils et choix méthodologiques privilégiés afin d'analyser les discours et les représentations des enseignants de notre corpus d'enquête.

- Un exposé succinct des résultats quantitatifs et qualitatifs de la recherche.

En conclusion, nous nous interrogerons sur les actions à envisager pour la mise en œuvre d'activités d'éveil aux langues en contexte scolaire dominiquais pour que tous les élèves puissent en bénéficier, quelque que soit leur répertoire verbal, leur origine ethnique, sociale ou culturelle.

\section{L'éveil aux langues}

En s'appuyant sur diverses recherches (notamment, le développement de l'éducation au plurilinguisme et à la diffusion des activités d'éveil aux langues au Canada et en France), Claude Cortier et Marisa Cavalli $(2013: 8)$ formulent les constats suivants :

" D'une manière générale, l'enseignement ou même seulement la prise en compte dans l'espace scolaire des langues qui ne sont pas les langues majeures de scolarisation n'est effective que dans peu de régions européennes, en dépit de politiques linguistiques incitatives dans ce domaine et/ou d'enseignements bilingues bien établis, comme c'est le cas dans certaines régions [...] (Catalogne, Corse, Val d'Aoste notamment) :

- les enseignants sont encore peu formés et peu armés, mais aussi très peu valorisés lorsqu'ils élaborent des démarches visant à accepter et utiliser en classe une ou des langues ou variétés qui ne sont pas la ou les variétés dominantes scolaires ;

- s'il s'agit d'un enseignement de langue régionale ou minoritaire, c'est souvent la variété standard (lorsqu'il y en a une) qui est privilégiée, sans prise en compte (ou trop peu) des autres variétés et le rapport à la norme (et l'hypercorrection souvent corrélée) se trouve alors fonctionner comme dans le cas des langues de scolarisation nationales.

On peut alors aussi observer en milieu scolaire des attitudes de mépris (sinon de déni) face à l'usage par les enfants / adolescents des formes locales, des régionalismes ou plus encore des formes de contact, hybrides, interlectes, parlers jeunes, qui témoignent de l'histoire et de l'actualité des langues et des sociétés [...].»

Face à de telles problématiques, l'éveil aux langues s'est révélé être une démarche adaptée et une expérience concluante pour répondre aux besoins et capacités des élèves (Kervran, 2013, 2011). Michel Candelier (2007: 371) rappelle, en quelques lignes, les tenants et aboutissants de cette approche qui a fait ces preuves, dès les années 1990, dans divers contextes sociolinguistiques (Allemagne, Autriche, France et Suisse romande) :

«L'éveil aux langues apparaît comme un moyen par lequel on veut (ou peut) courir plusieurs lièvres à la fois :

- développer l'intérêt et l'ouverture des élèves vis-à-vis de la diversité, y compris de la diversité propre; corollairement, dans des classes multilingues, reconnaître, légitimer et valoriser les compétences et identités linguistiques et culturelles de chacun ;

- développer l'aptitude des élèves à observer et analyser les langues, et donc favoriser leur aptitude à les apprendre et à mieux les maîtriser, y compris pour la langue de l'école ;

- favoriser le désir des élèves d'apprendre les langues, et d'apprendre des langues diversifiées ; 
- développer chez les élèves des connaissances relatives à la présence des langues dans l'environnement immédiat, plus lointain et très lointain, ainsi qu'aux statuts dont elles bénéficient ou pâtissent. »

À l'instar de ces projets européens, d'autres actions autour de l'éveil aux langues ont été entreprises en contexte créolophone, et plus spécifiquement en Guyane (Candelier, 2007). Avec plus d'une trentaine de langues inventoriées en Guyane, ce département français d'Outre-Mer est caractérisé par un degré élevé de multilinguisme dont les langues pèsent un poids (numérique, économique, symbolique, politique, etc.) significatif et soulèvent des enjeux sociaux, éducatifs et pédagogiques considérables (Alby et Léglise, 2014). Compte tenu des caractéristiques du contexte guyanais, Michel Candelier (2007:372) a préconisé un programme cherchant à conserver "l'unité dans la diversité » en s'appuyant sur les modalités d'une implantation d'un éveil aux langues, à savoir :

"-Du degré plus ou moins élevé de multilinguisme social ;

- du statut des langues qui constituent ce multilinguisme (langues autochtones, langues des migrants); de la présence plus ou moins répandue de la ou des langues officielles en tant que langue(s) de communication courante ;

- de son (leur) rôle en tant que langue de communication internationale; des moyens dont disposent les systèmes éducatifs ; des cultures éducatives elles-mêmes. »

Après avoir relevé les obstacles possibles à un enseignement bilingue en Guyane, Michel Candelier (2007 : 375-376) a souligné les avantages d'un éveil aux langues pour ce contexte :

"L'éveil aux langues est applicable à tous les types de classe, y compris linguistiquement très hétérogènes ;

- il exige des moyens matériels et financiers incomparablement plus réduits qu'un enseignement bilingue;

- il peut, dans une seule et même démarche [...] concerner toutes les langues en présence, indépendamment du nombre de leurs locuteurs et de leur statut;

- il exprime une reconnaissance par l'école de la langue de tous les élèves ; [...]

- en banalisant les situations de diglossie [...], il contribue au dépassement des antagonismes au sein de la classe et de la société ; [...]

- il contribue à développer la curiosité des enfants allophones à propos de leur propre langue [...] ; par le recours à une perspective comparatiste, il fournit à l'élève alloglotte quelques exemples de mise en relation entre sa propre langue et d'autres langues, dont la langue des apprentissages scolaires [...]. »

Dans quelle mesure la situation dominiquaise est-elle similaire à celle de la Guyane ? Quelles sont les caractéristiques de l'île? L'éveil aux langues peut-elle être une alternative appropriée?

\section{Le macro-contexte de la recherche : la Dominique dans tous ses états !}

Nous présenterons brièvement le contexte social, politique et économique de la Dominique. Puis, nous nous attarderons sur la situation sociolinguistique de l'île avant d'aborder l'approche glottopolitique qui en est faite, notamment en contexte scolaire. 


\subsection{Le contexte social, politique et économique : un état-nation en développement}

Le terme «Commonwealth of Dominica » est souvent utilisé pour distinguer la République de la Dominique de la République Dominicaine. Trait d'union entre deux départements français d'Outre-Mer - la Guadeloupe au nord et la Martinique au sud - la Dominique est un petit étatnation insulaire des Petites Antilles, avec ses cinquante kilomètres de long et vingt-quatre kilomètres de large pour une superficie d'environ $751 \mathrm{~km}^{2}$ (Murat, 2013). Roseau, la capitale, se situe sur la côte sud-ouest et compte à elle seule plus de $20 \%$ de la population active de l'île qui s'élève à 72301 habitants (Central Statistical Office, 2014). D'après le recensement de 2011, la tranche d'âge des 15-34 ans représente 31,1\% de la population dominiquaise (Central Statistical Office, 2014). Dans son ouvrage sur la Dynamique identitaire de la Dominique, Bruce Jno-Baptiste (2008 : 15) souligne que l'île connaît « une situation socio-économique assez vulnérable». Le secteur primaire (l'agriculture et la pêche notamment) représente " plus d'un tiers de la main d'œuvre dominiquaise » (Abd-Kadir et al., 2003 : 4). En août 1979, quelques mois seulement après l'accès à l'indépendance ${ }^{2}$, le passage sur l'île du cyclone David a fait des milliers de sans-abri et a entraîné une grave crise économique et sociale (Murat, 2013). L'émigration a constitué un exutoire et les transferts de fonds des émigrés ont représenté un apport important pour l'île, fréquemment frappée par des intempéries ${ }^{3}$ (Ibid.). Selon l'étude menée par la Caribbean Development Bank (2010), 29\% des foyers (représentant $40 \%$ de la population) vivent en situation de pauvreté et $10 \%$ des foyers sont en situation de pauvreté extrême. Entre 2009 et 2013, le taux de croissance du produit national brut (PNB) annuel était de -1,7\% (Caribbean Atlas, 2013) avec 20\% du PNB généré par le secteur primaire et $32,8 \%$ par le secondaire. La dépendance à l'importation de la quasi-totalité des produits de consommation, à l'exception de quelques produits agroalimentaires, ne diminue pas. L'île connaît ainsi un déficit commercial structurel important, trois fois plus qu'elle n'exporte. D'après le Caribbean Atlas (2013), les principaux partenaires d'importations sont les Etats-Unis : 36,8\% ; Trinité-et-Tobago : 17\% ; l'Union Européenne : $9 \%$; la Chine : $2,4 \%$ et le Japon : 2,4\%. Les déficits externes majeurs ne sont que partiellement compensés par le secteur tertiaire, soit 30\% du PIB avec les recettes touristiques, notamment lors du festival international de musique créole « World Creole Music Festival » qui a lieu au mois d'octobre.

Pour conclure, Bruce Jno-Baptiste (2008 : 21-22) souligne que pour des petits états-nations comme la Dominique, le terme « développement » convoque divers paramètres :
"Le développement est souvent compris à tort comme concernant la seule croissance économique. Pourtant, il met aussi en jeu la longévité, l'éducation, la liberté, le bonheur et d'autres facteurs qui améliorent la qualité de nos vies [...]. [La] compatibilité [entre les projets culturels et les projets de développement] est au cour de la préoccupation de la population [dominiquaise] qui voit se concrétiser à la fois ses aspirations culturelles et une espérance d'un mieux-vivre et donc d'un mieux-être si le pays parvient à tirer profit de ses ressources humaines et naturelles. »

Si le développement de la Dominique exige la participation de toutes les couches de la population, quelle politique adopter en matière de langues pour assurer le développement? Quelle est l'approche glottopolitique de la situation sociale et linguistique de l'île ?

\footnotetext{
${ }^{2}$ L'île accéda à l'indépendance le 03 novembre 1978 et devint une République parlementaire démocratique dans le Commonwealth.

${ }^{3}$ En 1989 et en 2007, les cyclones Hugo et Dean ont ravagé l'île et meurtri sa population.
} 


\subsection{La situation (socio)linguistique}

Louis Guespin (1985 : 21) a proposé de « substituer au terme de 'politique de la langue' celui de glottopolitique» pour rendre compte de tout ce qui concerne la "gestion des pratiques langagières»(Guespin, 1985: 23). La glottopolitique consiste à étudier «les diverses approches qu'une société a de l'action sur le langage, qu'elle en soit ou non consciente» en tenant compte de "tous les faits de langage où l'action de la société revêt la forme du politique » (Guespin et Marcellesi, 1986 : 5). Elle couvre alors les aspects micro- et macrosociolinguistiques, dans le sens où elle s'étend des interactions quotidiennes jusqu'aux interventions les plus globales du pouvoir politique sur les langues (planification juridique, système scolaire, entre autres). Quelles sont les langues qui véhiculent le patrimoine culturel de la Dominique? Quel statut occupent-elles au sein de la société ? Pour y répondre, nous proposons de présenter successivement la langue officielle, la langue dite nationale, les langues vernaculaires, les langues vivantes étrangères, et enfin, les langues d'immigration.

\subsubsection{La langue officielle : l'anglais}

La Constitution de la Dominique de 1978 ne fait mention que de la langue anglaise. En effet, l'article 31 de la Constitution oblige tout candidat à une élection parlementaire à être capable de lire l'anglais avec un degré de compétence suffisant pour lui permettre de participer activement aux mesures de la Chambre d'assemblée.

1) [...] a person shall be qualified to be elected as a Representative if, [...], he -

b) is able to speak and, unless incapacitated by blindness or other physical cause, to read the English language with a degree of proficiency sufficient to enable him to take an active part in the proceedings of the House.

2) Subject to the provisions of section 32 of this Constitution, a person shall be qualified unless, he - [...]

c) is able to speak and, unless incapacitated by blindness or other physical cause, to read the English language with a degree of proficiency sufficient to enable him to take an active part in the proceedings of the House. (Constitution of Dominica, 1978 : article 31)

Quant à l'article 8 de la Constitution, il stipule qu'une personne accusée d'un acte criminel sera informée de la nature de l'accusation dans une langue qu'elle comprend.

2) Every person who is charged with a criminal offence - [...]

b) Shall be informed as soon as reasonably practicable, in a language that he understands and in detail, [...]

f) Shall be permitted to have without payment the assistant of an interpreter if he cannot understand the language used at the trial. (Constitution of Dominica, 1978 : article 8)

Malgré cette ouverture affichée envers d'autres langues, l'anglais demeure la langue de l'État. C'est la langue des activités parlementaires, de la rédaction et de la promulgation des lois et des cours de justice. En tant que langue officielle, elle est aussi la langue de l'administration, des médias et le médium d'enseignement. Mais de quel anglais s'agit-il ? D'après Françoise Gadet (2007: 13), "il n'est pas de langue que ses locuteurs ne manient sous des formes diversifiées ", et ce, à tous les niveaux de la langue (phonologique, morphologique, syntaxique et lexicale). À la Dominique, il existe une variété de l'anglais appelée le «dominican Englishl'anglais dominiquais » (Christie, 1994). Le caractère local de cette langue lui confère souvent l'appellation de dialecte, sachant que toute langue en possède, sans exception (Grommes et $\mathrm{Hu}$, 
2014). Notons au passage que le dialecte est défini comme la variété ${ }^{4}$ régionale d'une langue alors qu'un patois est, à l'intérieur d'un dialecte, un parler spécifique à une région délimitée. À la Dominique, nous retrouvons le créole à base lexicale française ${ }^{5}$ qui est souvent désigné par les locuteurs comme un patois, et ce, sans connotation péjorative (Abd-Kadir et al., 2003).

\subsubsection{La langue dite nationale : le créole à base lexicale française}

Pour désigner le statut du créole à la Dominique, l'expression « langue nationale » est utilisée de facto pour nommer une autre langue qui n'est pas définie comme officielle par l'État car ce dernier ne s'engage pas à utiliser cette langue, mais seulement à assurer sa protection et sa promotion, puis d'en faciliter son usage par les citoyens. L'objet de cette mesure est de reconnaître que le groupe n'est pas une simple minorité : il fait partie du patrimoine national. En 1967, c'est cette reconnaissance qui a été attribuée au créole par le Premier ministre de la Dominique, Edward Oliver Le Blanc, lors de l'ouverture de la célébration de la journée nationale (St-Hilaire, 2011). En 1981, cette volonté politique s'est concrétisée par la création d'un centre d'études créoles connu sous 1'appellation KEK «Konmité pou Etid Kwéyòl »" (Paugh, 2005 : 1808). De telles initiatives ont été amorcées pour d'autres langues régionales ou minoritaires ${ }^{7}$ comme le garifuna et le kokoy.

\subsubsection{Les langues vernaculaires : garifuna et kokoy}

Langues vernaculaires $^{8}$, le garifuna et le kokoy viennent enrichir le paysage linguistique et culturel de la Dominique. Le premier est un héritage linguistique des descendants Caraïbes (De Pury, 2001). Depuis 1903, la plupart des Amérindiens de la Dominique - des Zambos (métis d'Indiens Caraïbes et de Noirs) - vivent dans une réserve forestière de 1800 hectares, située à $40 \mathrm{~km}$ de Roseau et au nord-est du pays (dans les villages de Concord, Bataca, Crayfish River, Salybia, Saint-Cyr, Gaulette River, Mahaut River et Sineku). Un maire - le «Carib chief ${ }^{9}-$ a été nommé pour représenter ces circonscriptions. D'après Simone Maguy Pézeron (1993), malgré la montée de la revendication identitaire de la communauté amérindienne, à travers notamment la création du Garifuna Cultural Group et la fondation d'un groupe politique défendant l'identité caraïbe, le Carib Liberation Movement, la langue garifuna ne jouit d'aucun statut officiel sur l'île. En outre, les recherches de Charlène WhiteChristian (2006) indiquent que la langue est en voie de disparition. Seules les personnes âgées

\footnotetext{
4 D'après Claude Cortier et Marisa Cavalli (2013: 111), la «variation peut être historique (diachronie), géographique (régiolecte), mais aussi et surtout sociale en fonction de l'âge et des niveaux sociaux (sociolecte) ou des professions (technolecte); elle est aussi individuelle (idiolecte). L'étude des variations [...] montre qu'une même personne parle différemment selon la situation de communication, l'interlocuteur, le registre (oral, écrit) et la fonction du discours $\gg$.

${ }^{5}$ La littérature emploie l'expression « créole à base lexicale ... » pour spécifier l'origine européenne de son lexique.

${ }^{6}$ Reporter et chroniqueur radio, Félix Henderson présente également le journal en créole (au Dominica Broadcasting Service Radio - 'DBS radio', créée en 1971) et préside en parallèle ce comité d'études créoles qui fait partie d'une délégation du ministère de la culture - le « Cultural Division » - et a pour but de promouvoir et de valoriser le créole.

${ }^{7}$ Nous utilisons indifféremment les expressions « langue nationale » et « langue régionale ou minoritaire » et retenons la définition de Claude Cortier et Marisa Cavalli (2013 : 208) qui s'appuie sur la charte européenne des langues régionales ou minoritaires (Conseil de l'Europe, 1992) : «- pratiquées traditionnellement sur un territoire d'un État par des ressortissants de cet État qui constituent un groupe numériquement inférieur au reste de la population de l'État; et - différente de la (des) langue(s) officielle(s) de cet État. Cette définition n'inclut ni les dialectes de la (des) langue(s) officielle(s) de l'État ni les langues des migrants ».

${ }^{8}$ C'est « une langue utilisée dans le cadre des échanges informels entre proches du même groupe, [...], quelle que soit sa diffusion à l'extérieur de ce cadre (qu'elle soit ou non véhiculaire). » (Calvet, 1997 : 292).

${ }^{9}$ Il dépend du ministère des affaires caraïbes « ministry of Carib affairs » (Chambers, $1994: 37$ ).
} 
continuent à le pratiquer (ci-après quelques exemples d'énoncés). La nouvelle génération accorde une importance plus significative à l'anglais et au créole à base lexicale française.

\begin{tabular}{|c|c|c|}
\hline Garifuna & Transcription phonétique & Français \\
\hline Itènia karahi ? & [itعniakasai] & Ca va bien \\
\hline Ennaï tàboua nà kou & [Enaitabuanaku] & Je vais dormir \\
\hline (mia) lamahàtina & [mialamahatina] & J'ai faim \\
\hline Makarahàtina & [makabahatina] & J'ai soif \\
\hline
\end{tabular}

Tableau 1 : Exemples d'énoncés en garifuna (White-Christian, 2006)

Le kokoy, un créole à base lexicale anglaise, n'échappe pas à ce manque de reconnaissance de la part du gouvernement dominiquais. Malgré les revendications et initiatives du comité national, le National Kokoy Council (Magloire-Akpa, 2010: 8), le kokoy ne jouit d'aucune reconnaissance officielle :

«Dans un effort de préserver et de promouvoir le kokoy comme langue populaire, la Division de la Culture lui donne une place assez importante dans les activités culturelles, surtout dans la célébration de l'Indépendance de la Dominique. On essaie également de réintroduire un programme hebdomadaire en kokoy qui existait auparavant à la radio nationale, Dominica Broadcasting Corporation. »

Il est principalement pratiqué dans le nord-est de l'île qui accueille des immigrés venus des îles anglaises voisines (Bryan et Burnette, 2003). Selon les travaux de Sonia Magloire-Akpa (2010 : 7), le kokoy serait aussi en voie de disparition :

«La langue maternelle ou langue seconde de quelques 8000 Dominiquais originaires des villages du nord-est de la Dominique. L'usage de cette langue est plus ou moins restreint à cette communauté dont quelques habitants âgés sont encore des locuteurs unilingues kokoy. Selon Margaret James dans un article intitulé «Keeping Kokoy Alive » dans l'hebdomadaire The Chronicle, le kokoy trouve ses origines dans le mouvement des esclaves libres des îles anglophones notamment Antigua, St. Kitts et Montserrat, au nord de la Dominique en 1871. Plusieurs familles ont quitté ces îles pour venir travailler sur les grands domaines comme Hatton Garden, Marigot, Melville Hall, Woodfordhill, et Londonderry au nord-est de la Dominique [...]. Il faut souligner qu'il y a très peu de locuteurs du kwéyol dans ces villages. Certains pensent que le kokoy est une langue en voie de disparition. D'après Arnold Telemacque, membre d'un groupe qui travaille au développement et à la promotion du kokoy, beaucoup de gens, surtout les jeunes ont oublié la langue car ils sont peu disposés à l'utiliser. »

À ce jour, il existe très peu de travaux sur le Kokoy de la Dominique (ci-dessous quelques exemples d'énoncés). Néanmoins, quelques projets ${ }^{10}$ de recherche ont permis par exemple d'étudier la dynamique lexicale et morpho-syntaxique de la langue (Aceto, 2010).

\footnotetext{
${ }^{10}$ Dans le cadre du Master Humanités créoles - Mention : Langues Littératures, Civilisations et CommunicationSpécialité Langues et Interculturalité préparé à l'Université des Antilles (et de la Guyane) sur le campus de Schœlcher entre 2006 et 2010, un cours sur l'étude du kokoy de la Dominique a été assuré par le lexicologue Bernadette Cervinka.
} 


\begin{tabular}{|c|c|c|}
\hline kokoy & Transcription phonétique & français \\
\hline Nor en nor hah non moe & [nวยnวanวmo] & Non, je n'en ai plus \\
\hline Mee mee wah gae ah praize tee day & [memewagoapseztidej] & Je vais à l'église aujourd'hui \\
\hline Mee pikni & [mipikni] & Mon enfant \\
\hline $\begin{array}{l}\text { Mee have fe go an work fee mee } \\
\text { family }\end{array}$ & [meavfigoanwokfimifamili] & $\begin{array}{l}\text { Je dois travailler pour subvenir aux } \\
\text { besoins de ma famille }\end{array}$ \\
\hline
\end{tabular}

Tableau 2 : Exemples d'énoncés en kokoy (White-Christian, 2006)

Les langues étudiées en contexte scolaire à la Dominique sont plutôt des langues venues d'ailleurs, à savoir: le français langue étrangère (désormais FLE) et l'espagnol langue étrangère.

\subsubsection{Les langues étrangères : français et espagnol}

Des langues de la Dominique aux langues en Dominique, nous identifions tout d'abord le français dont la pratique sur l'île est principalement circonscrite au contexte professionnel et familial, et ce, compte tenu de la proximité géographique avec les départements français d'Outre-Mer. Comme le souligne la correspondante nationale de la Dominique auprès de l'OIF ${ }^{11}$, Sonia Magloire-Akpa (2010 : 4), la communauté francophone n'est pas significative sur l'île :

«Le français reste pour la plupart des Dominiquais une langue étrangère, bien qu'elle soit parlée couramment par un faible pourcentage de la population, notamment des professeurs de français, des Français, d'autres francophones qui résident à la Dominique, des Dominiquais qui ont fait leurs études dans un pays francophone, certains fonctionnaires et les petits commerçants qui font du commerce avec les îles voisines de la Guadeloupe et de la Martinique. »

Pour assurer la promotion et la valorisation de la langue et de la culture françaises, la Dominique compte à son actif une alliance française et une Association des Professeurs de Langues Etrangères : le Dominican Association of Foreign Languages Teachers (DAFLT), entre autres. Selon la synthèse que fournit Philippe Girerd (2004 : 37) dans le rapport du premier colloque des Associations de professeurs de français dans les petites Antilles (O.E.C.S), l'intérêt général pour la langue et la culture françaises se heurte actuellement à "une forte campagne d'échanges de professeurs et de programmes de bourses d'études menées par les ambassades de Cuba et, dans une moindre mesure, aux cours gratuits offerts par les instituts vénézuéliens ». À Sonia Magloire-Akpa (2010:3) de souligner les rapports diplomatiques et la présence dans le nord de la Dominique de la diaspora dominicaine participant à la promotion de la langue et de la culture espagnoles sur l'île :

«La politique étrangère du gouvernement de la Dominique octroie une place importante aux relations diplomatiques avec l'Amérique Latine surtout avec Cuba et le Venezuela. En conséquence, de plus en plus de jeunes Dominiquais font leurs études supérieures à Cuba, au Venezuela et au Mexique, d'où ils reviennent avec une bonne maîtrise de l'espagnol. En plus, cette dernière langue devient un moyen de communication très important, surtout au nord de la Dominique où réside un nombre croissant d'immigrés venus de la République dominicaine. »

\footnotetext{
${ }^{11}$ L'OIF : Organisation Internationale de la Francophonie.
} 
D'autres langues - dites d'immigration - viennent compléter le tableau, à savoir : le chinois, le créole haïtien et le hindi.

\subsubsection{Les langues d'immigration : chinois, créole haïtien et hindi}

Depuis une décennie ${ }^{12}$, de nouveaux migrants viennent de pays très éloignés, notamment de la Chine. La présence grandissante du chinois est déterminée, d'une part, par le nombre d'étudiants chinois inscrits dans les cursus universitaires à la Dominique, et d'autre part, par les nombreux commerces chinois qui fleurissent dans la capitale. Depuis la rentrée scolaire 2013-2014, le chinois ${ }^{13}$ est également introduit dans les programmes scolaires d'une école secondaire privée catholique, St John's Academy ${ }^{14}$, située à Portsmouth. Par ailleurs, le rapport de Sonia Magloire-Akpa (2010:4) sur le «programme d'apprentissage du français en contexte multilingue - Dominique» avance qu'au «cours des dernières années, la Dominique a connu une forte immigration haïtienne, ce qui a introduit le créole haïtien dans le répertoire linguistique de la Dominique ». Dans notre recherche, nous avons également observé une pratique de la langue Hindi entre les petits commerçants de Roseau. Nous ne disposons pas d'étude fine sur les modalités de transmission de cette langue ni sur la façon dont elle coexiste avec les autres langues. Les locuteurs ne sont pas une composante importante du multilinguisme dominiquais, toutefois, quelle gestion scolaire est faite de la situation (socio)linguistique? Même si les langues véhiculaires dominantes à la Dominique sont le créole et l'anglais dominiquais, dans quelle mesure cette diversité linguistique et culturelle que nous venons d'exposer est-elle prise en compte à l'école ? Quelle est la politique linguistique éducative affichée, préconisée ou adoptée ? L'école favorise-t-elle l'égalité des chances et l'accès au savoir et à la culture à tous ?

\subsection{Les langues à l'école primaire : représentations, politiques et statuts des langues}

Dans la déclaration universelle de l'UNESCO sur la diversité culturelle (2001), cette dernière est considérée comme faisant partie du patrimoine commun de l'humanité qui, en tant que source d'échanges, d'innovation et de créativité est indispensable à l'humanité, au même titre que la biodiversité l'est à la nature. Il convient donc de la protéger pour le bien des générations actuelles comme des générations futures, et de la considérer comme l'un des droits fondamentaux de l'homme :

«La culture prend des formes diverses à travers le temps et l'espace. Cette diversité s'incarne dans l'originalité et la pluralité des identités qui caractérisent les groupes et les sociétés composant l'humanité. Source d'échanges, d'innovation et de créativité, la diversité culturelle est, pour le genre humain, aussi nécessaire que l'est la biodiversité dans l'ordre du vivant. En ce sens, elle constitue le patrimoine

\footnotetext{
${ }^{12}$ Le 24 mars 2014, les dirigeants de la Chine et de la Dominique se sont félicités mutuellement à l'occasion du $10^{\circ}$ anniversaire de l'établissement de leurs relations diplomatiques. Le président chinois Xi Jinping a rappelé au président dominiquais Charles Savarin comment ils ont fait avancer la coopération (matérialisée, par exemple, par la livraison en 2007 du stade national, le « Windsor Park National Sport Stadium » de 12000 places, situé à Roseau et entièrement financé par la Chine à hauteur de 12 millions d'euros (Jno-Baptiste, 2010).

${ }^{13}$ C'est Kim Douglas, fils de l'ancien Premier ministre de la Dominique Rosie Douglas, qui a assuré l'enseignement du chinois pour les élèves en Form 1 et 2. Alice Wang lui a succédé depuis la rentrée 2014.

${ }^{14}$ Officiellement ouverte depuis janvier 2013 avec une trentaine d'élèves, Juliana Magloire est la principale de la première école secondaire de la Dominique qui est complètement privée (dont l'inscription annuelle s'élève à 150 \$).
} 
commun de l'humanité et elle doit être reconnue et affirmée au bénéfice des générations présentes et des générations futures. »(UNESCO, 2001 : article 1)

Dans notre recherche, nous considérons alors la culture, dans une perspective anthropologique, comme une sorte de "répertoire de manières de penser et d'agir, commun à des individus, mais en permanence actualisé, renégocié, à travers la singularité de chacun » (Le Henaff, 2013 : 24). Sa prise en compte à l'école revêt dès lors une importance capitale, notamment dans la construction identitaire de l'enfant :

«Dans nos sociétés de plus en plus diversifiées, il est indispensable d'assurer une interaction harmonieuse et un vouloir vivre ensemble de personnes et de groupes aux identités culturelles à la fois plurielles, variées et dynamiques. Des politiques favorisant l'intégration et la participation de tous les citoyens sont garantes de la cohésion sociale, de la vitalité de la société civile et de la paix. Ainsi défini, le pluralisme culturel constitue la réponse politique au fait de la diversité culturelle. Indissociable d'un cadre démocratique, le pluralisme culturel est propice aux échanges culturels et à l'épanouissement des capacités créatrices qui nourrissent la vie publique. » (UNESCO, 2001 : article 2)

Par conséquent, nier ou dévaloriser la langue d'un élève équivaut à nier et à dévaloriser l'individu. Quel impact de telles considérations peuvent-elles avoir sur le développement de petits états-nations comme la Dominique ? Selon le Bulletin officiel de l'Éducation nationale $\mathrm{n}^{\circ} 33 \mathrm{du} 13$ septembre 2001, l'introduction des langues régionales ou minoritaires à l'école française "favorise la continuité entre l'environnement familial et social et le système éducatif, contribuant à l'intégration de chacun dans le tissu social de proximité », et ce, tout au long de la scolarité :

"L'article L. 312-10 du code de l'éducation a réaffirmé la possibilité de dispenser un enseignement des langues et cultures régionales tout au long de la scolarité dans les régions où celles-ci sont en usage. L'Éducation nationale se doit de faire vivre ce patrimoine culturel, de veiller au développement des langues régionales et de contribuer à leur transmission. Oublier cette responsabilité ne serait pas un signe de modernité. Ce serait au contraire une perte de substance de l'héritage culturel national. L'enseignement des langues et cultures régionales favorise la continuité entre l'environnement familial et social et le système éducatif, contribuant à l'intégration de chacun dans le tissu social de proximité. Cet enseignement s'applique actuellement au basque, au breton, au catalan, au corse, au créole, au gallo, à l'occitan-langue d'oc, aux langues régionales d'Alsace, aux langues régionales des pays mosellans, au tahitien, ainsi qu’aux autres langues mélanésiennes [...]. » (B.O. n³3, 2001)

Autrement dit, la langue n'est pas un moyen de communication neutre (Gasquet-Cyrus et Petitjean, 2009). C'est après avoir présenté le système éducatif dominiquais que nous présenterons la politique linguistique éducative que préconise celui-ci.

\subsubsection{Le système éducatif dominiquais : structure et fonctionnement}

Les premiers systèmes scolaires furent organisés au début du $19^{\text {ème }}$ siècle par les anglicans, les catholiques et les méthodistes. L'influence de ces églises est encore relativement marquée dans le fonctionnement interne du système scolaire (prière en début de classe, port des uniformes, entre autres). L'Éducation nationale est publique et est à la charge du gouvernement (même s'il existe des établissements privés sous contrat). Dans les deux 
cas, les parents contribuent au coût de la scolarité notamment par l'achat d'uniformes, de livres, de fournitures et des frais d'examens.

Le système scolaire est articulé à partir du modèle britannique (les niveaux de cycle primaire se déclinent en Grades) et du modèle nord-américain (les catégories dans le cycle secondaire s'articulent en Forms). La population scolaire est accueillie dans un système d'enseignement unifié, dont l'architecture générale est la suivante: enseignement primaire (maternelle et élémentaire), secondaire et supérieur. Il n'existe qu'un établissement post-secondaire, le «Dominica State College» à Roseau, qui réunit un institut universitaire de formation des maîtres (l'équivalent de l'ESPE ${ }^{15}$ ), une école d'infirmiers, un lycée académique et un lycée technique. Si l'on se réfère au texte de la Loi sur l'Education de 1997 (section 27 : 171) de la Dominique, la scolarité est obligatoire à partir de 5 ans jusqu'à l'âge de 16 ans :

\begin{abstract}
" Every child shall attend school from the first day of the school calendar in the school year following that in which he attains the lower age of the compulsory school age until the last day of the school calendar in the school year in which he attains sixteen years of age or at the end of which he obtains a school leaving certificate or diploma awarded by the school, whichever occurs first. " (Dominica Education Act 1997, $1998: 171)$
\end{abstract}

Aujourd'hui, dès trois ans, presque tous les enfants fréquentent l'école maternelle. Après ces années à l'école maternelle, les élèves entrent à l'école primaire dont les classes commencent par le Kindergarten puis Grade 1 à 6 . Dans la plupart des écoles, une semaine équivaut à vingt-cinq heures de cours. Dans la majorité des établissements scolaires, la journée ${ }^{16}$ de classe débute à huit heures et se termine à treize heures. Dans chaque école primaire (soit une soixantaine sur l'île), il y a un directeur d'école et chaque professeur est responsable d'une classe qui lui est attribuée. Le programme élémentaire aboutit à un examen commun «Common Entrance Examination » qui donne accès au niveau secondaire. Les résultats de ce test déterminent l'affectation des élèves dans les différents établissements secondaires. Les élèves qui obtiennent d'excellents résultats reçoivent une bourse de cinq ans, soit de la part du gouvernement, soit de la part des établissements privés (Magloire-Akpa, 2010). Quelle est la place des langues dans ce système éducatif, en particulier à l'école primaire ?

\title{
3.3.2. Politique linguistique éducative : quelle place pour quelle langue à l'école ?
}

C'est le français langue étrangère qui est enseignée dès le primaire. Depuis l'année scolaire 1995-1996, de la cinquième année du primaire (Grade 4) à la septième année (Grade 6), les élèves peuvent bénéficier d'une initiation à la langue et à la culture françaises d'une durée de trente à quarante-cinq minutes ${ }^{17}$ par semaine. Depuis quelques années, certaines écoles proposent aussi l'espagnol. Cette tendance tend à se répandre principalement dans les écoles situées au centre-ville, à Roseau. D'après le programme scolaire validé par l'Education Reform Unit (2008: 11), les principaux objectifs à atteindre en matière de langues au primaire correspondent au niveau A1 « utilisateur élémentaire »du CECRL ${ }^{18}$ (2001) :

\footnotetext{
${ }^{15}$ ESPE : École Supérieure du Professorat et de l'Éducation.

${ }^{16}$ Dans certains établissements, la journée s'étend parfois de neuf heures à quinze heures trente, avec une pause déjeuner entre midi et treize heures trente.

${ }^{17}$ Le chef d'établissement a la liberté de fixer le créneau horaire et la fréquence d'enseignement-apprentissage du français. En général, ce cours est supprimé lorsque les enseignants doivent boucler le programme scolaire.

${ }^{18}$ Le CECRL : Cadre Européen Commun de Référence pour les Langues.
} 
«1. Foster in the learner a positive attitude towards language in general and language learning in particular; 2. Help learners to become literate in the languages available to them; 3. Develop proficiency in Standard English while embracing / accepting and taking into account the child's first / home language and its usefulness in helping the child to learn; 4. Help the learner to become confident in the use of English, the primary language of instruction; 5. Enhance the learner's confidence, sense of identity and self-esteem through the use of language. " (OECS-OERU Education Reform Unit, 2008 : 11)

Aucune précision n'est apportée concernant la manière d'atteindre le premier objectif qui, notons-le au passage, s'inscrit tacitement dans une éducation au plurilinguisme. Quant au deuxième, il suppose que l'institution scolaire encourage une prise en compte des langues qui composent le répertoire verbal des élèves. Pourtant, dès l'école primaire, les objectifs évoluent en se focalisant exclusivement sur l'apprentissage des langues internationales comme le français et l'espagnol. Dans quelle mesure cette politique linguistique éducative influence-telle les représentations que les enseignants ont des langues et de leur apprentissage ? Quel discours portent-ils sur la diversité linguistique et culturelle à l'école primaire ?

\section{Les enseignants face aux langues : étude de cas}

Plusieurs outils et choix méthodologiques ont guidé notre présente recherche. À la question de savoir quelle approche méthodologique privilégier, Madeleine Grawitz (1986 : 174) rappelle que : " ce qui dicte le choix de la technique, c'est avant tout l'objectif de la recherche ». Sans plus tarder, exposons succinctement notre démarche empirique puis les résultats quantitatifs et qualitatifs de la recherche.

\section{1. Étude des discours et des représentations : outils et choix méthodologiques}

Pour qu'un objet devienne social, il doit être partagé par un ensemble d'individus (Abric, 2003). L'idée que les gens se font d'un objet social influence les rapports qu'ils entretiennent avec ce dernier; et parce que «le langage représente un certain type d'institution sociale 》 (Perrot, 1953 : 116), il n'échappe pas à cette règle : les gens s'en font une idée. Les représentations sociales sont des visions du monde qui se construisent dans l'interaction avec les autres car dans l'analyse qu'ils font des situations, les individus et les groupes introduisent toujours leurs nécessités subjectives. En effet, "les représentations sociales des uns ne sont pas celles des autres : elles reflètent un positionnement social (idéologique), inscrit dans une histoire (elles en sont le produit en même temps qu'elles participent à celles-ci)» (Moore et Py, 2008 : 275). En somme, les représentations instaurent "des versions de la réalité, communes et partagées » (Jodelet, 1989 : 49). Ce sont des « univers d'opinions» (Moscovici, 1961 : 66) propres à une culture, à un groupe et relatifs à des objets de l'environnement social. Comme le souligne Bernard Py (2004 : 6), le discours est «le milieu naturel par excellence des représentations sociales » car "c'est par le discours qu'elles existent et se diffusent dans le tissu social. En outre, le langage est un instrument très efficace de catégorisation ». Bien que l'approche linguistique des représentations à travers le discours soit une étape nécessaire voire incontournable, Marinette Matthey (1997: 320) attire notre attention sur le caractère ambivalent de ce rapport :

"Le discours n'est pas simplement un miroir reflétant les représentations, ou un écran transparent et perméable que l'on traverse pour atteindre un contenu cognitif. Pour le dire plus brièvement et d'une manière quelque peu dogmatique, le discours construit la réalité, il ne la reflète pas. [...] Or si nul ne nie l'importance du discours dans la 
formation et la transmission des représentations sociales, pour bon nombre de psychologues, le langage doit être traversé, voire déjoué, pour atteindre finalement le niveau purement cognitif de la représentation. [...] II semble bien que le langage peut faire écran à la représentation, que celle-ci peut se dissimuler derrière le langage. "

Autrement dit, le discours ne correspond pas à une représentation effective mais est un des éléments qui permet de la traiter. C'est pour cette raison que dans cette recherche, nous réserverons les termes "discours métalinguistiques » pour définir la capacité des enseignants à émettre une réflexion sur la langue et "discours épilinguistiques» pour désigner les sentiments et les jugements de valeur qu'ils portent sur la langue et qui se manifestent par des évaluations esthétiques ou des procédés de dénomination (le créole est un dialecte français, par exemple).

En somme, nous nous référons aux « représentations » pour rendre compte de la façon dont les enseignants pensent les langues et aux « discours » 19 pour rendre compte de la manière dont ils parlent des langues (en particulier du créole). À cette fin, nous avons mené une enquête $^{20}$ par questionnaire auprès de quarante-deux enseignants ${ }^{21}$ de FLE (soit $90 \%$ des enseignants de FLE de l'île). Six d'entre eux (tous des natifs et enseignants certifiés exerçant depuis plus de dix ans) ont accepté d'effectuer un entretien semi-directif ${ }^{22}$ dans le cadre de cette recherche-action de type exploratoire. Les cinq principales questions communes à ces deux outils méthodologiques étaient les suivantes: "parmi les langues que vous parlez, lesquelles considérez-vous comme les plus importantes ? »; « que pensez-vous de l'introduction du créole dans les programmes scolaires?»; «quel statut pourrait-il avoir?»; «son introduction aurait-il un impact positif sur l'enseignement en général ? »; et enfin, « quelles sont les langues présentes en classe de FLE? ».

\subsection{Résultats}

\subsection{1. Étude quantitative des données : l'analyse du questionnaire}

La majorité des enseignants (soit 59\%) déclare que parmi les langues qui composent leur répertoire verbal, l'anglais est la plus importante. Ces résultats ne sont pas surprenants compte tenu du statut officiel de la langue et des contraintes institutionnelles liées à la profession. Le français et la pratique commune de l'anglais et du créole se partagent la deuxième place (respectivement $18 \%$ et $19 \%$ ). Seule une minorité d'enseignants (soit $4 \%$ ) considère que toutes les langues se valent sans distinction de statut (internationale, régionale, minoritaire ou scolaire). Comme indiqué dans le graphique suivant, pour l'option "toutes : précisez...», aucun enseignant n'a souhaité restreindre son propos à quelques langues : toutes les langues sont importantes.

\footnotetext{
${ }^{19}$ Dans notre étude, l'analyse du discours tentera « de lier l'évidence sémantique à l'historicité du discours en s'appuyant à la fois sur l'intradiscours (le construit) et sur l'interdiscours (le préconstruit)» (Gafitescu, $2006: 123$ ).

${ }^{20}$ Entre 2007-2008, nous avons rencontré tous les enseignants de FLE lors des réunions trimestrielles avec la conseillère pédagogique du FLE à l'école des maîtres (ESPE) à Roseau. Ce travail s'inscrit dans un cadre plus large d'une étude comparée des pratiques et représentations sociales des langues en contexte scolaire plurilingue à la Dominique et à Sainte-Lucie (Cambrone-Lasnes, à paraître).

${ }^{21}$ Quarante enseignants sont des natifs et deux sont originaires des départements français d'Outre-Mer.

${ }^{22}$ Conventions de transcription : / pause courte (moins une seconde); // pause moyenne (1 à 2 secondes environ) et /// pause longue ( 2 à 3 secondes environ). Les interventions du chercheur marquées par la lettre $\mathrm{C}$ et $\mathrm{P}$ pour les professeurs.
} 


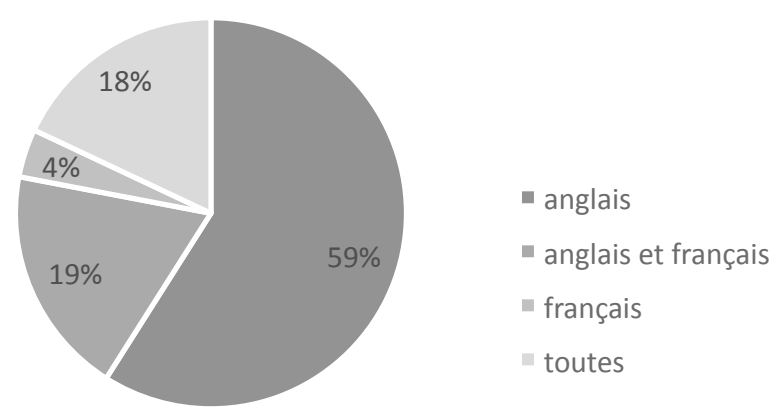

\section{Graphique 1 : Langues parlées les plus importantes}

Si les représentations sociales que les enseignants ont des langues les conduits à hiérarchiser ces dernières, que pensent-ils de l'introduction de la langue dite nationale dans les programmes scolaires ? Les avis sont mitigés. Si $12 \%$ y sont fortement opposés, la plupart est, soit très favorable (soit $40 \%$ ), soit sans un avis tranché sur la question (soit $48 \%$ ). En agrégeant ces données, on constate que la majorité des enseignants est suffisamment ouvert d'esprit pour envisager un programme scolaire qui tient compte de la réalité sociolinguistique : l'anglais n'est pas le seul vecteur de communication.

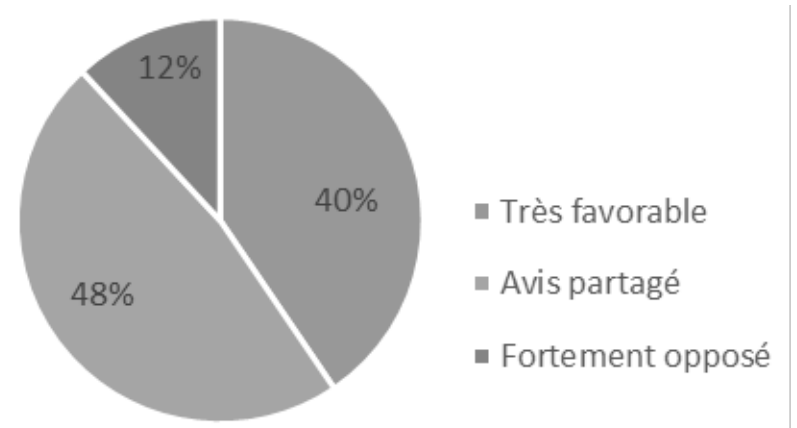

\section{Graphique 2 : Introduction du créole dans les programmes scolaires}

Le créole pourrait alors occuper plusieurs fonctions selon son statut à l'école. La majorité des enseignants (soit 43\%) envisage son introduction dans les programmes scolaires en tant que discipline optionnelle intégrée au programme déjà existant sur l'héritage culturel. Dans le cadre d'un projet d'enseignement bilingue, un quart des enseignants (soit 24\%) déclare qu'ils l'utiliseraient comme médium d'enseignement pour les apprentissages premiers. Pour d'autres (soit 28\%), il faudrait repenser les programmes en incluant le créole comme discipline à part entière afin d'aborder la langue sous tous ces aspects (linguistiques, culturels, pragmatiques et sociolinguistiques). Notons au passage que les chiffres indiquent une certaine cohérence dans les propos avec une minorité d'enseignants (soit 5\%) qui n'y voit aucun intérêt à son introduction à l'école voire qui s'oppose à tout statut éventuel. Dans quelle mesure un programme d'éveil aux langues pourrait-il fédérer ces différentes synergies pour favoriser la réussite scolaire des élèves et répondre aux enjeux éducatifs qui en découlent? 


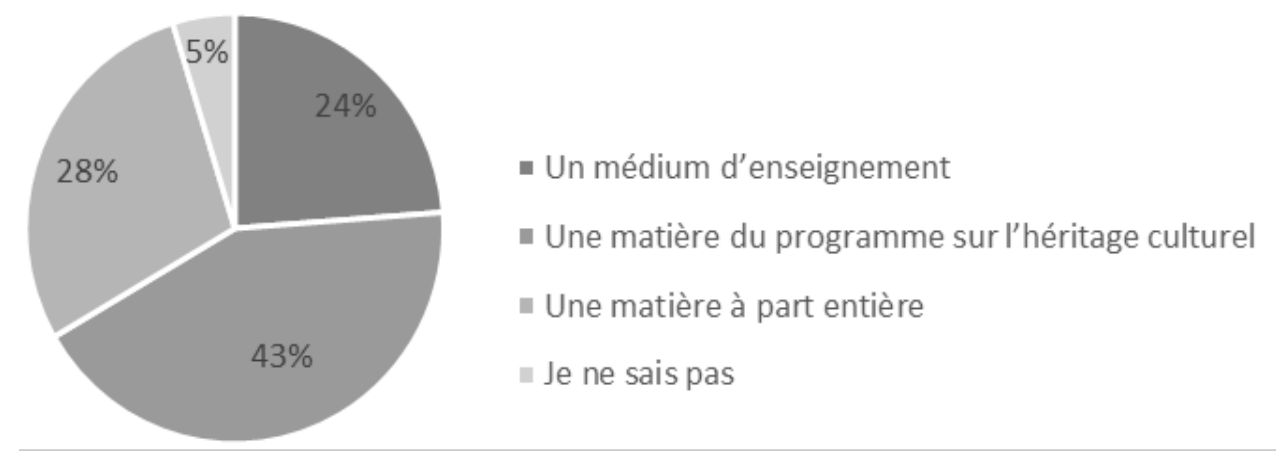

Graphique 3 : Le statut du créole à l'école primaire

Remettre en question la prééminence de l'anglais en contexte scolaire dominiquais - en y incluant le créole - conduirait, selon $67 \%$ des enseignants, à influencer positivement et significativement l'enseignement de façon générale. À cet égard, un tiers (soit $31 \%$ ) nuance le propos en estimant que l'impact positif ne serait pas conséquent. D'autres (soit 2\%) formule un avis plus radical considérant que l'introduction du créole à l'école ne peut avoir aucun impact : ni sur la reconnaissance par l'école de la langue de tous les élèves, ni sur la contribution au dépassement des antagonismes au sein de classe et de la société, par exemple. En définitive, retenons que la majorité des enseignants de FLE à l'école primaire est favorable à une éducation au plurilinguisme impliquant aussi bien des langues internationales que des langues minoritaires comme le créole.

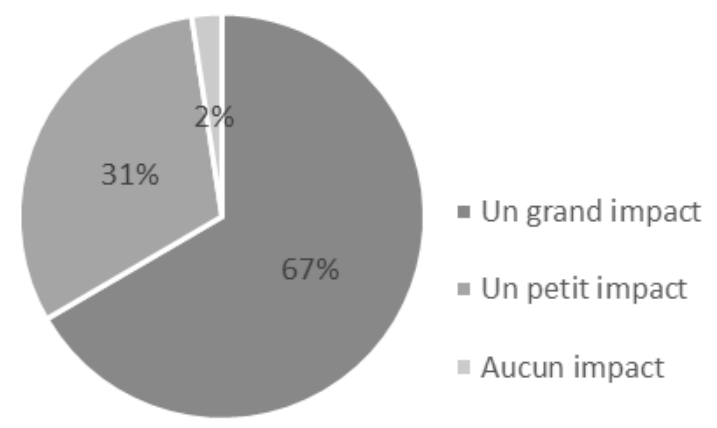

\section{Graphique 4 : Impact positif du créole sur l'enseignement en général}

Enfin, nous avons souhaité mesurer la corrélation entre l'impact escompté sur l'enseignement en général et les pratiques enseignantes en classe de FLE. Le moins que l'on puisse dire, c'est que les réponses sont concordantes. Si la majorité des enseignants (soit 71\%) déclare n'utiliser que l'anglais en classe de FLE, un quart (soit 24\%) affirme avoir recourt à une autre langue que la langue de scolarisation, à savoir le créole. D'autres (soit 5\%) stipulent qu'ils n'emploient que le français. Comment interpréter ce dernier résultat ? Notre connaissance du terrain dominiquais nous conduit à envisager les pistes suivantes (sans écarter une mauvaise interprétation de la question posée) :

- 1. Le degré élevé d'intercompréhension entre le français et le créole dominiquais, la proximité linguistique entre ces langues et le phénomène de l'alternance codique en classe ne permettent pas à ces enseignants de distinguer leur utilisation effective de ces langues en classe ;

- 2. Certains enseignants considèrent que le créole est un dialecte français et que lorsqu'ils parlent le créole dominiquais, c'est comme s'ils pratiquaient, dans une certaine mesure, le français. 
Ces résultats sont donc révélateurs, d'une part, des représentations que les enseignants ont des langues et de leur apprentissage, et d'autre part, des représentations qu'ils ont de leurs propres pratiques professionnelles.

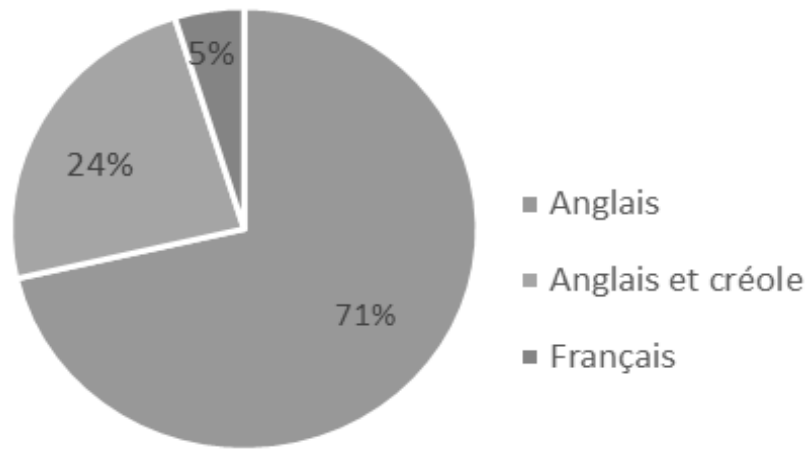

\section{Graphique 5 : Utilisation des langues en classe de FLE}

En définitive, plusieurs langues semblent être présentes en classe de FLE. Quelles sont leurs fonctions respectives? Livrent-elles des pistes de réflexion pour la mise en œuvre d'activités d'éveil aux langues en contexte dominiquais? En d'autres termes, toutes les langues à l'école : pourquoi et pour quoi faire? L'étude qualitative des données à travers l'analyse des entretiens semi-directifs s'attachera à éclaircir ces différents points en identifiant les enjeux sous-jacents.

\subsection{2. Étude qualitative des données : l'analyse des entretiens semi-directifs}

Dans sa réflexion sur la dynamique sociale et culturelle des langues créoles dans la Caraïbe, Paulette Durizot Jno-Baptiste (2001 : 10) se pose la question suivante :

«Peut-on faire apparaître plus clairement l'identité culturelle des Caribéens à travers leurs représentations des langues européennes? Il nous importe de savoir si le discours identitaire varie selon la dynamique des langues européennes et créoles dans les trois sociétés d'étude: Curaçao (Caraïbe néerlandaise), Dominique (Caraïbe anglaise) et l'archipel guadeloupéen (Caraïbe française). ”

À l'instar de cette recherche, notre étude des discours et des représentations des enseignants de FLE nous a permis de répertorier trois enjeux majeurs liés à la prise en compte de la diversité linguistique et culturelle à l'école primaire en contexte dominiquais : des enjeux culturels, éducatifs et pédagogiques. Par ailleurs, le discours des enseignants est souvent paradoxal et révélateur d'une représentation diglossique de l'enseignement-apprentissage des langues. Alors que le créole est décrit par un des témoins (P1) comme la langue d'appartenance, sa langue maternelle, la langue véhiculaire, la langue de cœur, ce dernier exclut par là même son utilisation en contexte scolaire pour éviter de perturber les élèves dans leur apprentissage de l'anglais et du français, langues qui sont suffisamment " compliquées ». De plus, le créole est une langue à tradition orale qui n'a pas besoin d'être apprise ou étudiée sous sa forme écrite. Sa transmission intergénérationnelle, dans le cadre familial et extrascolaire, assure sa pérennité. Par ailleurs, le créole ne répondrait à aucune règle linguistique stricte, ce qui rendrait son apprentissage d'autant plus facile :

3.C: Ok so why Creole is not then officially introduced?

3.P1: If Creole is introduced in schools where it is not inherent / it does not have to be part of the curriculum / it simply needs to be encouraged among teachers and students and it will catch on // Creole was not written or taught at school but it 
became so well rooted that educationists thought that there was an urgent need to subdue Creole in order that the development of Standard English would not be hindered // this probably helped to a certain extent but creole was newer subdued // even those who were flogged or punished for speaking it in and out of school still learned and passed it on to their children // Creole is the language which is learned easily because of its absence of strict structural components // it is an effective means of communication which may be used for explanation and audiovisual demonstration // pupils can learn creole at home / on the street and anywhere // it doesn't need to be written // as it might hinder the learning of Standard French

4.C: $\quad$ So why should Creole be introduced at schools?

4.P1: Creole should be encouraged in schools as it is a language of the heart / a language of emotion / a language of socialization / a language of expression // my mother was fluent in English / my father could only speak Creole / he could not read and write // my mother used to read stories in English and explain them to us in Creole // we were raised in Creole // values were transmitted to us through Creole // we did not have to read and write but we learned the language fluently // Creole can be encouraged at home // I would encourage government to teach Creole at school and children to learn through drama speech debates / general knowledge quizzes in Creole competition but not to write and structure Creole // English and French are complicated enough / children should not be given the additional challenge of learning a grammar and structure for Creole

Ces enjeux interrogent donc la formation des enseignants de FLE à et par la diversité. D'après les principes du CECRL (2001 : 129) qui inscrivent l'enseignement-apprentissage des langues dans une perspective actionnelle, ce qui est en jeu ici c'est :

"Une vision " cloisonnante » de la / des compétence(s) des individus en matière de langues et de cultures, [...] : cette compétence ne consiste pas en " une collection de compétences à communiquer distinctes et séparées suivant les langues » mais bien en une " compétence plurilingue et pluriculturelle qui englobe l'ensemble du répertoire langagier à disposition. "

Dans la perspective d'une éducation au plurilinguisme, ces enseignants de FLE pourraient alors être sensibilisés aux savoirs, savoir-faire et attitudes suivants (Castellotti, Coste et Duverger, $2008: 20-21)$ :

"Savoir que les langues appréhendent la réalité de manière conventionnelle, symbolique et arbitraire; qu'il existe des différences de fonctionnement entre les langues, les discours, les modes de communication, etc. ; avoir des connaissances sur les relations entre langues, cultures, sociétés; savoir qu'il existe une grande diversité linguistique et culturelle; savoir que la variation est constitutive des langues; savoir mobiliser ses connaissances et expériences linguistiques pour en aborder de nouvelles; savoir tirer parti de situations nouvelles pour réfléchir en retour sur les connaissances antérieures et les modifier; savoir comparer les phénomènes linguistiques / langagiers ; savoir utiliser ce que l'on sait dans une/plusieurs langues pour comprendre ou produire dans une autre langue; savoir tenir compte du répertoire de ses interlocuteurs et des caractéristiques sociolinguistiques pour communiquer efficacement et à bon escient; savoir percevoir/reproduire des éléments linguistiques non familiers; avoir une attention et une sensibilité à la diversité et à la variation; être curieux et ouvert vis-à-vis des différences linguistiques et culturelles; accepter l'altérité linguistique et culturelle; accepter de modifier ses certitudes linguistiques et culturelles; être disposé à s'engager 
dans l'apprentissage de nouvelles langues; prendre conscience de la pluralité des identités dans son entourage. »

D'autres témoins (P2, entre autres) poursuivent la réflexion en rappelant qu'à la Dominique, c'est la tradition d'un enseignement monolingue qui domine, et ce, compte tenu de la mondialisation. De ce fait, le futur citoyen dominiquais se caractérise par une capacité à s'adapter à une diversité de situations de communication tout en se forgeant une identité créole :

14.C : Pensez-vous que le gouvernement / les parents et les enseignants encourageraient un projet scolaire incluant les langues parlées à la Dominique?

$14 . P 2$ :Non / à mon avis ils encourageront ce que j'ai dit / c'est-à-dire qu'ils voudraient que l'anglais soit le plus important comme il est la langue plus marquée au monde commercial et avec la globalisation et que le créole soit comme un parcours culturel pour créer des futures citoyens éduqués dans tous aspects de notre culture // [...]

9.C: Que pensez-vous du créole en tant médium d'instruction? Y seriez-vous favorable?

9.P2 : Absolument pas // le créole ne peut pas être en compétition avec l'anglais qui est la langue plus populaire dans le monde / alors pour préparer nos étudiants l'anglais doit être premier

10.C: Alors si le créole devait être introduit à l'école, quel statut devrait-il avoir?

10.P2 : As a core component forming part of their cultural heritage programme / this is best as again Creole can't compete with universally popular languages as French and Spanish

Enfin, quelques enseignants (P1, P2 et P5, entre autres) ont attiré l'attention sur la diversité de leurs pratiques enseignantes pour répondre principalement à des enjeux éducatifs (accueil des enfants allophones) et pédagogiques (ne pas occulter la langue maternelle de l'enfant pour faciliter l'accès aux apprentissages qu'il soit issu des milieux ruraux et/ou urbains) :

17.C : Est-ce que les élèves parlent le créole en classe de français?

$17 . P 2$ :Rarement je rappelle un seul exemple avec un étudiant qui parle créole / avec un étudiant haïtien quand il a commencé l'école à la Dominique / il était l'un d'un petit nombre des élèves qui pouvait parler créole / alors le créole l'a beaucoup aidé jusqu'à ce qu'il puisse communiquer en anglais // quelques fois les élèves utilisent le créole pour amplifier un message comme une commande au lieu de dire " shut up » en anglais qui est poli / on dit " pé bouch ou » qui est plus fort I la plupart de temps ce technique marche.

2.C: What do you think of the introduction of Creole at school?

2.P1 : Introducing Creole at school is a good thing / in my opinion / Creole is already introduced in many schools not as a core subject on the curriculum but as a medium to explain concepts in various subject areas / where students with limited experiential backgrounds encounter difficulty in understanding concepts and terminologies used // sometimes all it takes is for the teacher to use a few words or phrases in creole and the learning becomes clear.

3.C: In your opinion in what language should the learners be taught to fully grasp the concepts taught? 
3.P5 : Both in English and Creole because there is a disparity between the children of today and yesterday / children of urban and suburban areas do not understand creole as much as children from the rural areas / I think the teaching in both English and Creole should be implemented only in those areas where there are Creole-speaking students and where the teachers also have a good command of the Creole language.

En définitive, privilégier une éducation plurilingue au détriment d'un enseignement cloisonné des langues se traduit par deux orientations majeures sur le terrain de l'école primaire (Castellotti, Coste et Duverger, 2008 : 20-21) :

«- Une conception globale de l'éducation aux langues [...] qui intègre l'enseignement et l'apprentissage de toutes les langues et variétés, mais aussi une réflexion décloisonnée sur les phénomènes de la "sphère linguistique ", se développant de manière trans- et interlinguistique ;

- Une prise en compte opératoire de la transversalité de la dimension linguistique / langagière dans toutes les composantes du curriculum scolaire. La finalité première étant le développement d'une compétence permettant de "fonctionner " (travailler, communiquer...) adéquatement, efficacement et en connaissance de cause dans un environnement pluriel, en termes de langues, mais aussi de modes d'expression, oraux, écrits et non verbaux.»

Quelles sont les pistes de réflexion qui peuvent ainsi être menées en ce sens à la Dominique ?

\section{Conclusion}

$\mathrm{Au}$ terme de cette enquête exploratoire, nous avons identifié des enjeux culturels, éducatifs et pédagogiques liés à la prise en compte de la diversité linguistique et culturelle à l'école primaire en contexte dominiquais. Ces derniers sont tributaires de facteurs d'ordres sociolinguistiques (répertoire verbal des locuteurs), professionnels (formation des enseignants à une didactique du plurilinguisme) et individuels (représentations des langues).

En nous appuyant sur les premiers résultats de notre enquête de terrain et les modalités d'implantation d'un éveil aux langues, nous pouvons avancer qu'un tel programme peut être envisagé en contexte dominiquais, notamment afin d'une part, de "contribuer à développer la curiosité des enfants allophones à propos de leur propre langue» et "contribuer au dépassement des antagonismes au sein de la classe et de la société», et d'autre part, pour " reconnaître, légitimer et valoriser les compétences et identités linguistiques et culturelles de chacun » (Candelier, 2007 : 376). Le moins que l'on puisse dire, c'est que pour un petit étatnation comme la Dominique, la prise en compte de la diversité linguistique et culturelle à l'école peut constituer un facteur important pour son développement « durable ».

Pour tenter de conclure, il est judicieux de rappeler que les arguments souvent avancés pour justifier d'une éducation en faveur d'une diversité linguistique et culturelle (Castellotti, Coste et Duverger, 2008 : 17) sont les suivants :

«- Tous les enfants scolarisés possèdent déjà un répertoire langagier diversifié [...].

La structuration langagière que l'école opère ne peut ignorer cet état de fait.

- D'autant que les images de soi en tant qu'apprenant importent pour la réussite scolaire ; elles se construisent et se détruisent très tôt et dépendent aussi de la 
manière dont l'école accueille ou ignore les ressources et l'expérience linguistiques et culturelles que, dans leur diversité, possèdent les enfants.

- C'est au moment où les repères individuels se mettent en place que le contact et l'interaction avec l'altérité permettent l'affirmation des identités. [...]

- C'est à ce niveau que la réflexion sur la langue, et plus particulièrement la langue de scolarisation, ne peut que bénéficier d'une exposition à la variation et à d'autres fonctionnements linguistiques (ce qui pose le problème des outils d'un travail métalinguistique intégré). "

Enfin, un projet pilote est actuellement à l'étude afin d'initier un programme d'éveil aux langues en contexte scolaire dominiquais en collaboration avec la coordinatrice nationale des langues étrangères au ministère de l'Éducation de la Dominique, Charlène White-Christian.

\section{Références bibliographiques}

Abd-Kadir, J., Hardman, F. et Blaize, J. (2003). Dialect interference in the writing of primary school children in the Commonwealth of Dominica. Dans L1 - Educational Studies in Language and Literature (p. 1-14). Netherlands : Kluwer Academic Publishers.

Abric, J.-C. (dir.). 2003. Méthodes d'études des représentations sociales. Ramonville-Saint-Agne : Erès.

Aceto, M. (2010). Dominican Kokoy. Dans D. Schreier et al. (dir.), The Lesser-Known Varieties of English. An introduction (p. 171-194). Cambridge : Cambridge University Press.

Alby, S. et Léglise, I. (2014). Pratiques et attitudes linguistiques des enseignants. La gestion du plurilinguisme à l'école en Guyane. Dans I. Nocus, J. Vernaudon, et M. Paia (dir.), L'école plurilingue en Outre-Mer. Apprendre plusieurs langues, plusieurs langues pour apprendre : l'école plurilingue en Outre-Mer (p. 245-268). Rennes : Presses Universitaires de Rennes.

Berger, P.-L. (1997). Four faces of Global Culture. The National Interest, 49, $23-29$.

Bryan, B. et Burnette, R. (2003). Language variation and language use among teachers in Dominica. Dans M. Aceto et J. P. Williams (dir.), Contact Englishes of the Eastern Caribbean, Varieties of English Around the World G30 (p.141-154), Amsterdam : John Benjamins.

Bulletin Officiel de l'Education Nationale. (13 septembre 2001), 33, France : Éducation nationale.

Calvet, L.-J. (1997). Vernaculaire. Dans M.-L. Moreau, (dir.), Sociolinguistique, Concepts de base (p. 291-292). Bruxelles : Mardaga.

Cambrone, S. (2008). Pratiques langagières dans les Antilles françaises: le phénomène de l'alternance codique. Dans F. Calixte et E. Dorismond (dir.), Langues et éducation dans la Caraïbe (p. 107-124). Paris : L'Harmattan.

Cambrone-Lasnes, S. (à paraître, sous presse). Pratiques et représentations sociales des langues en contexte scolaire plurilingue. Etude comparée de la Dominique et de Sainte-Lucie (préfacé par Geneviève Zarate). Collection Transversales, Berne : Peter Lang.

Cambrone-Lasnes, S. (à paraître). L'éveil aux langues comme activité périscolaire à l'école maternelle : des pratiques aux attitudes linguistiques des enfants en région parisienne. Dans $\mathrm{S}$. Cambrone-Lasnes, A.-B. Kruger et N. Thamin (dir.), Diversité linguistique et culturelle à l'école primaire : accueil des élèves et formation des acteurs (préfacé par Danièle Moore). Revue Carnets d'ateliers de sociolinguistique. Paris : L'Harmattan.

Cambrone-Lasnes, S., Kruger, A.-B. et Thamin, N. (dir.) (à paraître). Diversité linguistique et culturelle à l'école primaire : accueil des élèves et formation des acteurs (préfacé par Danièle Moore). Revue Carnets d'ateliers de sociolinguistique. Paris : L'Harmattan. 
Candelier, M. (dir.). (2007). CARAP. Cadre de Référence pour les Approches Plurielles des Langues et des Cultures, Recherche et Développement du CELV. Strasbourg : Conseil de l'Europe.

Caribbean Atlas. (2014). Dominique. Martinique: Ceregmia-Université des Antilles et de la Guyane.

Caribbean Atlas. (2013). Dominique. Martinique: Ceregmia-Université des Antilles et de la Guyane.

Caribbean Development Bank. (2010). Country Poverty Assessment - Dominica, Final report. Trinidad and Tobago : Kairi Consultants Limited.

Castellotti, V., Coste, D. et Duverger, J. (dir.) (2008). Propositions pour une éducation au plurilinguisme en contexte scolaire. Paris : ADEB.

Central Statistical Office. (2014). Population and Housing Census. Roseau : Government of the Commonwealth of Dominica.

Chambers, C. (1994). Sociocultural Characteristics of the Dominican Caribs. Results of a Focus Group Study. Roseau : Ministry of Community Development and Social Affairs.

Christie, P. (1994). Language Preference in Two Communities in Dominica, West Indies. La Linguistique, 30, 7-16.

Conseil de l'Europe (2001). Cadre Européen commun de références pour les langues: apprendre, enseigner, évaluer. Unités des Politiques linguistiques. Strasbourg: Conseil de l'Europe.

Conseil de l'Europe (1992). La Charte européenne des langues régionales ou minoritaires. Série des traités européens, 148. Strasbourg : Conseil de l'Europe.

Constitution of the Commonwealth of Dominica. (1978). Roseau : Commonwealth of Dominica.

De Pury, S. (2001). Le garifuna, une langue mixte. Dans Langues de diaspora, langues de contact. Collection Faits de Langues (p. 75-84), 19. Paris : Ophrys.

Dominica Education Act 11 of 1997. (1998). Commonwealth of Dominica.

Durizot Jno.-Baptiste, P. (2001). Cultures et stratégies identitaires dans la Caraïbe. Paris : L'Harmattan.

Gadet, F. (2007). La variation sociale en français, nouvelle édition revue et augmentée. Paris : Ophrys.

Gafitescu, M. (2006). Henri Boyer, De l'autre côté du discours. Recherche sur les représentations communautaires. Mots. Les langages du politique, 80, 121-123.

Gasquet-Cyrus, M. et Petitjean, C. (dir.) (2009). Le poids des langues. Dynamiques, représentations, contacts, conflits. Collection Espaces discursifs. Paris : L'Harmattan.

Girerd, P. (2004). Synthèse. Dans Rapport du premier colloque des associations de professeurs de français dans les petites Antilles (OECS) (p.36-41). Roseau: Alliance française de la Dominique.

Grawitz, M. (1996). Méthodes des sciences sociales. Paris : Dalloz.

Grommes, P. et $\mathrm{Hu}$, A. (dir.) (2014). Plurilingual education. Policies - practices - language development. Hamburg Studies on Linguistic Diversity, 3. Amsterdam : John Benjamins.

Guespin, L. (1985). Matériaux pour une glottopolitique. Cahiers de Linguistique Sociale, 7, 13-32.

Guespin L. et Marcellesi, J.-B. (1986). Pour la glottopolitique. Langages, 83, 5-34.

Hélot, C. et Rubio, M. N. (dir.) (2013). Développement du langage et plurilinguisme chez le jeune enfant. Toulouse : Erès.

Jno-Baptiste, B. (2010). Identité et développement dans le petit Etat de la Dominique : le rôle de la diaspora. Études caribéennes, 16. Disponible sur : http://etudescaribeennes.revues.org/4654

Jno-Baptiste, B. (2008). La dynamique identitaire de la Dominique - Quelles stratégies pour un petit Etat caribéen anglophone? Collection Recherches et documents Amériques latines. Paris : L'Harmattan.

Jodelet, D. (1989). Folies et représentations sociales. Collection Sociologie d'aujourd'hui. Paris : PUF. 
Kervran, M. (2013). Les langues du monde au quotidien : une approche interculturelle : cycle 1. Rennes : CRDP de Bretagne.

Kervran, M. (2011). Les langues à l'école primaire : quelle formation pour les enseignants ? Les langues modernes, 3.

Le Henaff, C. (2013). L'anglais à l'école élémentaire : analyse didactique de l'articulation entre la langue et la culture. Thèse de doctorat non publiée. Rennes: Université de Rennes 2.

Magloire-Akpa, S. et al. (2010). Guide du maître : La Dominique. Dans R. Chaudenson, Adaptation de la didactique du français aux situations de créolophonie. Programme d'apprentissage du français en contexte multilingue. France : Organisation Internationale de la Francophonie.

Matthey, M. (dir.) (1997). Les langues et leurs images. Actes du colloque international. Neuchâtel : IRDP.

Moore, D. et Py, B. (2008). Introduction : Discours sur les langues et représentations sociales. Dans G. Zarate, C. Kramsch et D. Lévy (dir.), Précis du plurilinguisme et du pluriculturalisme (p. 275-283). Paris : Éditions des archives contemporaines.

Moscovici, S. (1961). La Psychanalyse, son image et son public. Paris : PUF.

Murat, C. (2013). Tourisme et lutte contre la pauvreté : les premiers enseignements de l'expérience de l'île de la Dominique (Petites Antilles). Études caribéennes, 24-25. Disponible sur: http://etudescaribeennes.revues.org/6530

OECS-OERU Education Reform Unit. (2008). Curriculum harmonisation. Dominica : Office of Educational Reform.

Paugh, A. L. (2005). Multilingual Play: Children's Code-Switching, Role Play, and Agency in Dominica, West Indies. Language in Society, 34, 63-80.

Perrot, J. (1953). La Linguistique. Paris : PUF.

Pezeron, S. M. (1993). The Carib Indians of Dominica Island in the West Indies: Five Hundred Years after Columbus. New York : Vantage press.

Simon, D.-L. et Maire Sandoz, M.-O. (2008). Faire vivre et développer le plurilinguisme à l'école : Les biographies langagières au cœur de la construction d'identités plurielles et du lien social. Dans M. Baurens et G. Martino (dir.), Langues-cultures à l'école primaire: quelle diversité pour quelle cohérence ? Études de linguistique appliquée (p. 265-276), 151. Paris : Didier Érudition.

St-Hilaire, A. (2011). Kwéyòl in Postcolonial Saint Lucia. Globalization, language planning, and national development. Amsterdam / Philadelphia : John Benjamins.

UNESCO. (2001). Déclaration universelle de l'UNESCO sur la diversité culturelle. Paris: UNESCO.

White-Christian, C. (2006). Le kwéyol dans la classe de FLE en Dominique. Les effets du créole dans l'acquisition et dans l'enseignement du Français Langue Etrangère. Dans D. Barreteau (dir.), L'enseignement du créole aujourd'hui. Etat des lieux, méthodes, pratiques et représentations (p. 113-123). Martinique : Edition IRD Martinique-Centre Caraibe. 